\title{
Sepsis and hyperoxia effects on the pulmonary surfactant system in wild-type and iNOS knockout mice
}

\author{
T.C. Bailey*, C. Cavanagh*, S. Mehta", , J.F. Lewis*,\#, R.A.W. Veldhuizen*,\#
}

\begin{abstract}
Sepsis and hyperoxia effects on the pulmonary surfactant system in wild-type and iNOS knockout mice. T.C. Bailey, C. Cavanagh, S. Mehta, J.F. Lewis, R. A.W. Veldhuizen. C ERS Journals Ltd 2002.

ABSTRACT: Alterations of pulmonary surfactant and increases in inducible nitric oxide synthase (iNOS) have been implicated in the pathophysiology of acute lung injury. It was hypothesised that these two observations are related and that alterations of the endogenous surfactant, due to either sepsis or hyperoxia, would be reduced in mice lacking the iNOS gene compared to wild-type mice.

Wild-type and iNOS (-I-) mice were randomised into sham or sepsis, and in a separate experiment animals were randomised to normoxia or hyperoxia exposure for $48 \mathrm{~h}$. Lungs were lavaged and analysed for total surfactant levels and surfactant subfractions (large (LA) and small (SA) aggregates).

Both sepsis groups had decreased SA compared to sham groups with no significant difference between the two genotypes. Mice exposed to hyperoxia had a decreased amount of total surfactant when compared to normoxia controls and there was no significant difference between the two genotypes.

It is concluded that inducible nitric oxide synthase does not influence the amount of pulmonary surfactant or surfactant subfractions recovered in lavage after $18 \mathrm{~h}$ of sepsis or 48 h of hyperoxia.

Eur Respir J 2002; 20: 177-182.
\end{abstract}

Depts of *Physiology, ${ }^{*}$ Medicine, and Pharmacology \& Toxicology, Lawson Health Research Institute, University of Western Ontario, London, ON, Canada.

Correspondence: T.C. Bailey, Lawson Health Research Institute, H417, 268 Grosvenor Street, London, ON, Canada, N6A 4V2.

Fax: 5196466110

E-mail: tbailey2@uwo.ca

Keywords: Acute lung injury, nitric oxide, pulmonary surfactant

Received: August 282001

Accepted after revision: January 18 2002

The present study was supported by the Dept of Medicine, University of Western Ontario and the Canadian Institute of Health Research.
Pulmonary surfactant reduces the surface tension within the alveoli and maintains lung compliance [1]. There are two aggregate forms that comprise the alveolar surfactant: large aggregates (LA) and small aggregates (SA). LA contain surfactant-associated proteins and are the surface-active component of the surfactant [2]. SA are the metabolic products of LA and are less surface active [3].

Alterations of the pulmonary surfactant system contribute to lung dysfunction in acute lung injury (ALI). A consistent alteration of surfactant observed in injured lungs is an increase in the relative amounts of SA with a concurrent decrease in LA subtypes [4]. Surfactant subtypes were also altered in the lungs at earlier stages of lung injury, prior to physiological abnormalities [5]. Although it was proposed that these surfactant alterations played a role in the development of lung injury, the specific changes at earlier stages of lung injury do not involve increases in SA and deceases in LA. For example, in septic rats with minimal physiological abnormalities, LA pool sizes remained the same as in sham but the SA pools were significantly reduced $[5,6]$. The mechanisms responsible for early changes in surfactant subfractions may be dependent on the specific insult leading to the lung dysfunction.

Increases in the expression and activity of inducible nitric oxide synthase (iNOS) have also been proposed to occur early during the development of, and contribute to, lung injury [7]. iNOS activity can be upregulated in response to various inflammatory mediators resulting in the production of a relatively large quantity of nitric oxide (NO) for prolonged periods of time. This NO may have a variety of effects on the lung, pathophysiological including vasodilation and increased vascular permeability [8, 9], as well as homeostatic including the regulation of inflammation [10]. Which specific process contributes most is unclear, but, like surfactant, NO effects may be dependant on the severity and nature of the injury.

Interestingly, based on a number of in vitro studies, one proposed deleterious effect of iNOS-derived NO is through alterations of the surfactant system [11-14]. Therefore, the purpose of this study was to examine the role of iNOS on the alveolar surfactant system at an early stage of lung injury in two mouse models of lung injury. It was hypothesised that the alterations of endogenous surfactant aggregates due to sepsis or hyperoxia would be reduced in mice lacking iNOS compared to wild-type animals.

\section{Materials and methods}

\section{General animal procedures}

Wild-type C57B1/6 mice (Charles River Laboratory, St-Constant, PQ, Canada) and previously 
characterised iNOS (-/-) (Jackson Labs, Bar Harbour, ME, USA) mice weighing 18-25 g, were used in these experiments [15]. The mice were group housed and allowed free access to standard rodent chow and water with a controlled $12 \mathrm{~h}$ light/dark cycle. After a minimum of 4 days acclimatisation these animals were used for either sepsis or hyperoxia experiments, as described below. The animal use subcommittee of the University of Western Ontario approved all procedures.

\section{Sepsis}

Of the wild-type mice 16 were randomised to the sham group and 21 to the sepsis group. Nine iNOS (-/-) mice were randomised into the sham group and 11 iNOS (-/-) mice were similarly randomised into the sepsis group. Mice were anaesthetised with a $0.6-\mathrm{mL}$ intraperitoneal injection $\left(50 \%\right.$ Ketamine: $100 \mathrm{mg} \cdot \mathrm{mL}^{-1}$; 5\% Xylazine: $20 \mathrm{mg} \cdot \mathrm{mL}^{-1}$; and $45 \%$ saline). The sepsis surgery involved performing a laparotomy and a cecal ligation and perforation (CLP) in which the cecum was exposed and ligated distal to the ileocecal valve, punctured twice with an 18-gauge needle and gently manipulated to exude a small amount of faecal material. The cecum was placed back into the abdomen, which was closed with 4.0 silk suture. Sham groups consisted of animals undergoing the anaesthetic procedures only. All animals then received a subcutaneous (s.c.) injection of buprenorphine $\left(0.04 \mathrm{mg} \cdot \mathrm{kg}^{-1}\right)$ in $1.5 \mathrm{~mL}$ of saline for analgesia and fluid resuscitation and were transferred to individual cages. All animals subsequently received a s.c. injection of $1.5 \mathrm{~mL}$ of saline every $6 \mathrm{~h}$ with an additional dose of buprenorphine $\left(0.04 \mathrm{mg} \cdot \mathrm{kg}^{-1}\right)$ at the $12 \mathrm{~h}$ time point. These injections were required for analgesia and maintenance of blood pressure, particularly in the sepsis animals. At the $18 \mathrm{~h}$ time point all mice were sacrificed as described below.

\section{Hyperoxia exposure}

Mice of both genotypes were randomised into either a $21 \%$ oxygen $\left(\mathrm{O}_{2}\right)$ exposed group (normoxia) and a group exposed to $>90 \% \mathrm{O}_{2}$ (hyperoxia). There were 11 mice in each group with the exception of the hyperoxia-exposed iNOS (-/) group, which comprised of nine mice. A sealed plexi-glass box $(55 \times 50 \times$ $32.5 \mathrm{~cm}$, Parker Plastics, London, ON, Canada) was filled with the appropriate gas mixture. The mice were placed inside the box and exposed for $48 \mathrm{~h}$ to either $\mathrm{O}_{2}$ concentration, with a gas flow rate of $10 \mathrm{~L} \cdot \mathrm{min}^{-1}$. $\mathrm{O}_{2}$ concentrations were controlled via a gas mixer (model 3500HL; Sechrist Medical Products Division, Anaheim, CA, USA), which received gases from the hospital's medical air and $\mathrm{O}_{2}$ lines. $\mathrm{O}_{2}$ levels (MiniOx-I oxygen analyser; MSA Medical Products, Pittsburgh, PA, USA) were verified every $12 \mathrm{~h}$.

\section{Analyses}

After the experimental procedures (18 h for sepsis group and $48 \mathrm{~h}$ for hyperoxia group), mice received an intraperitoneal injection of $0.5 \mathrm{~mL}$ of sodium pentabarbitol $\left(65 \mathrm{mg} \cdot \mathrm{mL}^{-1}\right)$ and a midline sternotomy was preformed. Plasma samples were obtained from blood aspirate from the left ventricle of the heart and snap frozen in liquid nitrogen until further use. Subsequently, the animal was exsanguinated via transection of the dorsal aorta. A tracheotomy was performed and an endotracheal tube was secured in place with 2.0 surgical silk. The lung was then carefully removed and lung compliance was assessed by static pressure/volume curves as previously described [16].

\section{Lung lavage procedure and analysis}

Following the compliance measurements a bronchoalveolar lavage was performed with $3 \times 1-\mathrm{mL}$ aliquots of $0.15 \mathrm{M} \mathrm{NaCl}$ saline [16]. Each lavage consisted of instilling the saline and withdrawing it repeatedly for an additional two times. The recovered lavages were combined and the total volume was recorded. The lavaged material was spun at $150 \times g$ for $10 \mathrm{~min}$ to remove cellular debris. The $150 \times g$ supernatant was stored at $-20^{\circ} \mathrm{C}$ except for a $1 \mathrm{~mL}$ aliquot which was used to separate the LA from the SA via centrifugation at $40,000 \times g$ for $15 \mathrm{~min}$. The $40,000 \times g$ pellet (the LA-fraction) was resuspended in $300 \mu \mathrm{L} 0.15 \mathrm{M}$ $\mathrm{NaCl}$ and frozen at $-20^{\circ} \mathrm{C}$ until further use. The SA fraction was also stored at $-20^{\circ} \mathrm{C}$.

Phospholipid-phosphorus measurements were used to determine the amount of surfactant in the $150 \times g$ supernatant and in the isolated surfactant subfractions. Aliquots of the different samples were extracted as described by BLIGH and DYeR [17]. The phospholipid-containing chloroform-phase was utilised for determination of phosphorus using a modification of the method by DUCK-CHONG [18]. A Lowry protein assay was used to measure protein concentrations in the $150 \times g$ supernatant [19].

\section{Nitric oxide metabolite analysis}

The NO metabolites, nitrites and nitrates (NOx), were analysed as described by WEBERT et al. [20]. Plasma samples were used for the sepsis experiment to examine systemic levels of NO metabolites. Three additional animals, per group, were used to collect nonlavaged lung homogenate samples to measure NO metabolites in the lung, a direct target of hyperoxia. Briefly, lungs were manually separated from airways and cardiac tissue and homogenised in buffer $(\mathrm{pH} 7.4$ with Hepes buffer $10 \mathrm{mM}$ : ethylenediamine tetraacetic (EDTA) at $0.1 \mathrm{mM}$, dithiothreitol $1 \mathrm{mM}$ and sucrose $0.32 \mathrm{M})$. The homogenate was then spun at $10,000 \times g$ for $10 \mathrm{~min}$ and the supernatant was removed, sealed under nitrogen and frozen. The NOx assay was performed using a chemiluminescence analyser.

\section{Surface activity measurements}

Separate mice, wild-type only, were used for a comparison of the surface activity of the surfactant of 
normoxia to hyperoxia surfactant. LA were pooled from 4 mice per pool with 3 pools per group and diluted in $150 \mathrm{mM} \mathrm{NaCl}, 2 \mathrm{mM}$ TrisHCl, $1.5 \mathrm{mM}$ $\mathrm{CaCl}_{2}(\mathrm{pH} 7.0)$ to a concentration of $250 \mu \mathrm{g} \cdot \mathrm{mL}^{-1}$ phosphorous. A captive bubble surfactometer was used to determine surface activity of the pooled LA samples as previously reported [21]. The data for adsorption to equilibrium is expressed in time and the minimum surface tension is expressed in milli Newton/meter $(\mathrm{mN} / \mathrm{m})$.

\section{Statistical analysis}

All data is presented as mean \pm SEM. All statistical comparisons were determined by one-way analysis of variance with a Tukey post-hoc analysis. A $\mathrm{p}<0.05$ was considered to be statistically significant.

\section{Results}

\section{Sepsis}

Animal data for the mice utilised in the sepsis experiment are shown in table 1. All sham mice survived the entire $18 \mathrm{~h}$ whereas sepsis in the wildtype mice resulted in death in six of the 21 animals. Systemically induced multiorgan failure was the probable cause of these deaths. The sepsis iNOS (-/-) mice also had increased mortality compared to the sham group with three of the 11 animals not surviving the $18 \mathrm{~h}$. Only animals surviving the 18 -h period were included for surfactant analysis. At the time of sacrifice none of the animals had a significant change in body mass. The volumes obtained from the lavage procedure were not different among the four groups. Analysis of static pressure/volume curves after sacrifice indicated no significant differences among the four groups (data not shown).

Comparison of the two wild-type groups revealed that sepsis animals had significantly $(\mathrm{p}<0.05)$ higher plasma NO metabolite (NOx) values than the sham group (table 1). The sepsis iNOS (-/-) mice and the sham iNOS (-/-) mice had similar plasma NOx values (table 1). Comparisons between the two genotypes revealed that the sepsis-wildtype group had significantly greater plasma NOx levels than the sepsis iNOS

Table 1.-Summary of animal masses, mortality, total lavage volumes and plasma nitric oxide metabolites (NOx) levels from the sepsis study

\begin{tabular}{lcccc}
\hline Group & Mass g & Mortality & $\begin{array}{c}\text { Total lavage } \\
\text { volume mL }\end{array}$ & $\begin{array}{c}\text { Plasma } \\
\text { NOx } \mu M\end{array}$ \\
\hline Sham-wt & $23.1 \pm 0.5$ & $0 / 16$ & $2.2 \pm 0.1$ & $27.4 \pm 3.5$ \\
Sepsis-wt & $22.5 \pm 0.5$ & $6 / 21$ & $2.3 \pm 0.1$ & $40.7 \pm 4.3^{*}$ \\
Sham-ko & $21.5 \pm 0.8$ & $0 / 9$ & $2.4 \pm 0.1$ & $14.1 \pm 2.1$ \\
Sepsis-ko & $21.7 \pm 0.4$ & $3 / 11$ & $2.2 \pm 0.1$ & $8.8 \pm 1.2$ \\
\hline
\end{tabular}

Data are presented as means \pm SEM. Sepsis: laparotomy and cecal ligation perforation group; wt: wild-type mice; ko: inducible nitric oxide synthase (-/-) mice. $*$ : $\mathrm{p}<0.05$ versus all.

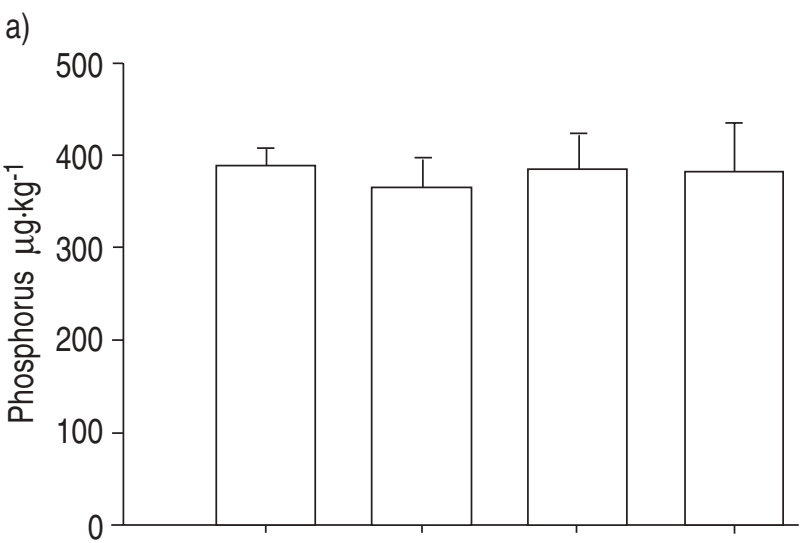

b)

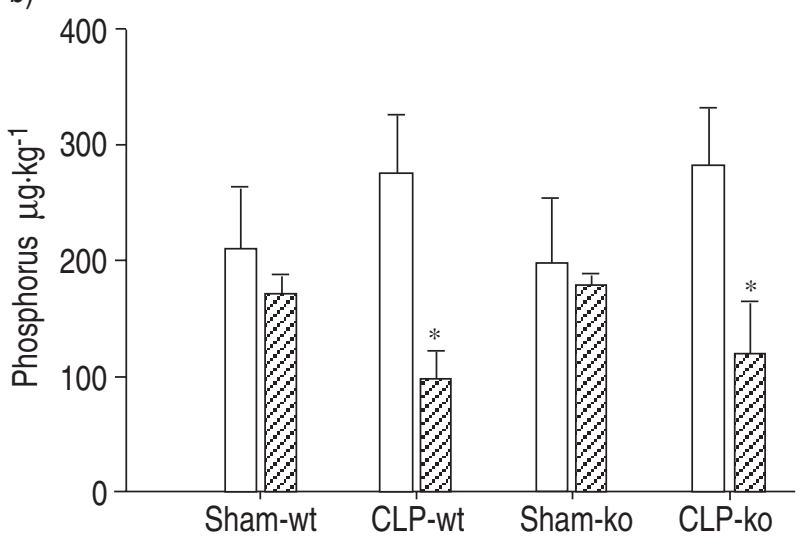

Fig. 1.-Analysis of phosphorous measured in lavage from sham and cecal ligation and perforation (CLP) groups. a) Total phosphorous in sham wild-type (Sham-wt: $n=8)$, CLP wild-type (CLP-wt: $n=8)$, sham inducible nitric oxide synthase (iNOS) (-/-) (Sham-ko: $n=8)$ and CLP iNOS (-/-) (CLP-ko: n=8). b) Phosphorous in large aggregates $(\square)$ and small aggregates $(\mathbb{Z})$. All data are presented as mean \pm SEM. *: $\mathrm{p}<0.05$ versus sham.

(-/-) group whereas the two sham groups had similar plasma NOx values.

The total amount of surfactant recovered in the lavage (fig. 1a) was not significantly different among the four groups. The amount of LA (fig. 1b) was higher in the two sepsis groups compared to their respective sham groups, however, these differences did not reach statistical significance. SA values obtained from the sepsis wild-type animals were significantly lower than values obtained from sham wild-type animals. Similarly, the amount of SA in the sepsis iNOS (-/) animals was significantly lower than in the sham iNOS (-/-) group. Comparison of the wildtype animals to the iNOS (-/-) groups revealed no significant differences between either the two sham groups or the two sepsis groups.

In order to allow for comparisons among different studies, the surfactant subfraction data were also expressed as the percentage of LA (\%LA). These data revealed a significantly higher \%LA in the sepsis wild-type group when compared with the sham wild-type group ( $73 \pm 5$ versus $51 \pm 5 \%$, respectively). The sepsis iNOS (-/-) group also had a significantly higher percentage of LA compared with the iNOS (-/-) group ( $71 \pm 6$ versus $47 \pm 5 \%$, respectively). There was 
Table 2. - Summary of animal masses, mortality, total lavage volumes and lung homogenate nitric oxide metabolites (NOx) from the hyperoxia study

\begin{tabular}{lcccc}
\hline Group & Mass g & Mortality & $\begin{array}{c}\text { Total } \\
\text { lavage } \mathrm{mL}\end{array}$ & $\begin{array}{c}\text { Lung } \\
\text { NOx } \mu \mathrm{M}\end{array}$ \\
\hline Norm-wt & $20.1 \pm 0.5$ & $0 / 11$ & $2.3 \pm 0.1$ & $6.6 \pm 1.0$ \\
Hyp-wt & $19.2 \pm 0.4$ & $0 / 11$ & $2.4 \pm 0.1$ & $10.6 \pm 3.7 *$ \\
Norm-ko & $20.3 \pm 0.3$ & $0 / 11$ & $2.5 \pm 0.1$ & $2.8 \pm 0.3$ \\
Hyp-ko & $20.5 \pm 0.3$ & $0 / 9$ & $2.3 \pm 0.1$ & $3.5 \pm 0.8$ \\
\hline
\end{tabular}

Data are presented as mean \pm SEM. norm: normoxia exposed; hyp: hyperoxia exposed; wt: wild-type mice; ko: inducible nitric oxide synthase (-/-) mice. ${ }^{*}: \mathrm{p}<0.05$ versus norm-ko.

no significant difference between wild-type and the iNOS (-/-) groups.

The total amount of protein recovered in the lavage was not different among all four groups. These values, corrected for bodyweight, were $23.8 \pm 2.6 \mu \mathrm{g} \cdot \mathrm{g}^{-1}, 21.6 \pm$ $4.0 \mu \mathrm{g} \cdot \mathrm{g}^{-1}, 33.6 \pm 4.3 \mu \mathrm{g} \cdot \mathrm{g}^{-1}$ and $13.0 \pm 4.0 \mu \mathrm{g} \cdot \mathrm{g}^{-1}$ for sham wild-type, sepsis wild-type, sham iNOS (-/-) and sepsis iNOS (-/-) respectively.

\section{Hyperoxia exposure}

There were no changes in bodyweight during the $48 \mathrm{~h}$ of exposure to either room air or hyperoxia and there was no mortality in any of the experimental groups (table 2). There were also no significant differences among the four groups in the recovered lavage volumes (table 2) or in the pressure/volume curves obtained after sacrifice (data not shown). For the hyperoxia experiment, NOx levels were measured in the homogenised lung tissue rather than plasma (table 2). For wild-type animals higher NOx concentrations were observed in the hyperoxia mice when compared with the normoxia, however this difference did not reach statistical significance. Overall, iNOS (-/-) animals had lower, but not statistically different, tissue NOx levels than the wild-type mice.

The total amount of surfactant recovered in the lavage was significantly lower $(p<0.05)$ in the hyperoxia wild-type groups compared to the normoxia wild-type group (fig. 2a). Similarly, for the iNOS (-/-) animals, the hyperoxia mice had significantly $(p<0.05)$ lower amounts of surfactant than the normoxia group (fig. 2a). There were no significant differences between the two mice genotypes. Values for the surfactant subfractions are shown in figure $2 \mathrm{~b}$. The hyperoxia wild-type mice had significantly less LA and SA compared to the normoxia wild-type group. The hyperoxia iNOS (-/-) group had a lower level of LA and SA compared to the normoxia iNOS (-/-) group, however, this was statistically significant only for the SA samples (fig. 2b). Comparisons between the wild-type and iNOS (-/-) groups revealed no significant differences. Expressing the data as \%LA showed similar values for all four groups.

As shown in table 3, the normoxia wild-type animals had a significantly $(\mathrm{p}<0.05)$ lower amount of protein compared to the hyperoxia wild-type group. In contrast, the normoxia iNOS (-/-) group
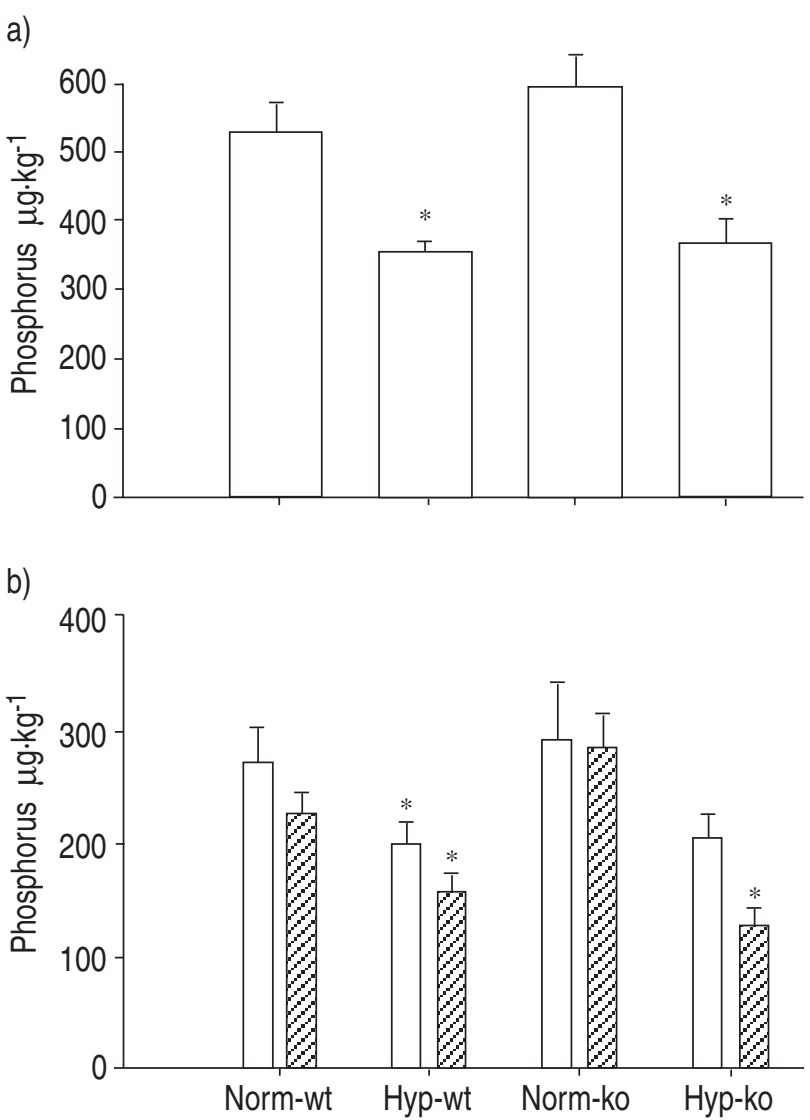

Fig. 2.-Analysis of phosphorous measured in lavage from normoxia and hyperoxia groups. a) Total phosphorous in normoxia wild-type (Norm-wt: $n=8$ ), hyperoxia wild-type (Hyp-wt: $n=8$ ), normoxia inducible nitric oxide synthase (iNOS) (-/-) (Norm-ko: $\mathrm{n}=8)$ and hyperoxia iNOS $(-/-)$ (Hyp-ko: $n=6)$. b) Phosphorous in large aggregates $(\square)$ and small aggregates $(\mathbb{Z})$. All data are presented as mean \pm SEM. $*: \mathrm{p}<0.05$ versus normoxia.

had similar amounts of protein as the hyperoxia iNOS (-/-) group (table 3). Normoxia wild-type animals and the normoxia iNOS (-/-) mice were not statistically different. Protein values in the hyperoxia wild-type group were higher than the hyperoxia iNOS (-/-) group; however this difference did not reach statistical significance.

Separate wild-type animals were used to collect pooled LA samples for the surface activity measurements. There was no statistically significant difference in the time for the LA to adsorb to an equilibrium surface tension $(6 \pm 2 \mathrm{~min}$, and $4 \pm 1 \mathrm{~min}$ for normoxia

Table 3.-Total amount of protein in bronchoalveolar lavage (BAL)

\begin{tabular}{llc}
\hline Group & $\mathrm{n}$ & Total protein in BAL $\mu \mathrm{g} \cdot \mathrm{g}^{-1}$ \\
\hline Norm-wt & 4 & $17.7 \pm 5.0$ \\
Hyp-wt & 8 & $45.0 \pm 6.3^{*}$ \\
Norm-ko & 5 & $22.8 \pm 2.1$ \\
Hyp-ko & 4 & $28.7 \pm 2.1$ \\
\hline
\end{tabular}

Data are presented as mean \pm SEM. norm: normoxia exposed; hyp: hyperoxia exposed; wt: wildtype-mice; ko: inducible nitric oxide synthase (-/-) mice. *: $\mathrm{p}<0.05$ versus Norm-wt. 
and hyperoxia respectively). After five cycles of quasistatic compressions the minimum surface tension for the normoxia samples was $12.8 \pm 2.3 \mathrm{mN} \cdot \mathrm{m}^{-1}$ and $11.2 \pm 3.7 \mathrm{mN} \cdot \mathrm{m}^{-1}$ for the hyperoxia samples.

\section{Discussion}

Alterations in pulmonary surfactant and increased endogenous NO production have both been implicated in the development of ALI and acute respiratory distress syndrome $[4,7,22]$. It was hypothesised that these changes may be related and that the increases in NO due to iNOS may impact the pulmonary surfactant system. This hypothesis was tested by examining alveolar surfactant from wild-type and iNOS (-/-) mice in two different models of lung injury that are known to alter endogenous surfactant. Under the present control conditions (sham and normoxia, respectively) there were no differences between these two genotypes. Similarly, the results showed no difference between the two genotypes in the experimental conditions. Previous studies have demonstrated that the iNOS (-/-) mice did not have compensatory responses from other nitric oxide synthase isoforms [23, 24]. Thus, the conclusions from the present experiments are that in the two models of lung injury, iNOS did not influence the amount of pulmonary surfactant or the amount of surfactant subfractions recovered in the lavage.

The authors focused specifically on the surfactant pool sizes since this change appears to be one of the first signals of surfactant alterations in the development of lung injury $[4,5]$. In the sepsis experiment, changes in surfactant aggregates occurred before any detectable physiological abnormalities or alterations of surfactant function at this time point [5, 25]. Similarly, in the hyperoxia model, 48-h exposure resulted in a change in surfactant pools but no detectable change in lung compliance or in the activity of isolated LA. Thus, both experimental models utilised in this study represent relatively early stages in the development of lung injury. Although not measured in this study, increases in iNOS expression/ activity have been reported during the course of sepsis [24] and during $48 \mathrm{~h}$ of hyperoxia in mice [26] utilising the same conditions and similar strains of mice as the current experiments. These previous observations were indirectly supported by the NOx results.

The specific effects of the two models on the pulmonary surfactant system were slightly different suggesting that the injury in these two models develop differently. Sepsis induces a focal point of infection that progresses to a systemic inflammatory response. The lung is one of the targets of this increased systemic response leading to the observation of decreased SA [5]. This observation prompted the current authors to investigate factors that are induced in the lung by the systemic inflammation that subsequently may impact upon the surfactant system. Since the results indicate that iNOS is not directly involved in producing a decrease in SA following sepsis, further studies are required to elucidate the exact mechanisms by which the sepsis procedure affects the surfactant system.

In contrast to sepsis, exposure to hyperoxia causes a more direct insult to the lung via increased oxidative stress. The concept of increased iNOS adversely affecting the surfactant system in this model was based on the knowledge that reactive $\mathrm{O}_{2}$ species produced during hyperoxia could generate peroxynitrate resulting in nitrosylation of tyrosine residues on surfactant protein (SP)-A and/or other surfactant proteins, thereby causing an altered alveolar metabolism [25, 27, 28]. Based on the results here the current authors speculate that iNOS has little direct, or indirect via SP-A, effect on surfactant metabolism.

Interestingly, in hyperoxia the increase in lavage protein concentrations in the hyperoxia wild-type animals was attenuated in the hyperoxia iNOS (-/-) animals. This result indicates that iNOS contributed to the increased permeability caused by hyperoxia and confirms reports in other models of lung injury. Although increased lavage protein does not represent an alteration of surfactant, it may have implications for surfactant function since inhibition of surfactant by serum proteins is a well-known mechanism of surfactant dysfunction [29]. In the current model of hyperoxia, however, the increase in lavage protein, although statistically significant, was still relatively small and did not yet impact the surface activity of isolated LA.

The alterations of surfactant at early stages of lung injury contrast with the surfactant alterations in severely injured lungs. Surfactant from patients with acute lung injury has an altered lipid composition, decreased surfactant proteins and reduced amounts of large aggregates [30, 31]. These changes, combined with a large influx of inhibitory proteins, result in a significant decrease in surfactant activity, which contribute to lung dysfunction. It is possible that increased inducible nitric oxide synthase activity contributes to the severe surfactant alterations observed in acute lung injury rather than at the earlier stages of lung injury.

Acknowledgements. The authors would like to thank F. Possmayer for the use of the captive bubble tensiometer, D. McCormack for valuable discussion and $\mathrm{S}$. Weicker for technical assistance.

\section{References}

1. Possmayer F, Yu SH, Weber JM, Harding PG. Pulmonary surfactant. Can J Biochem Cell Biol 1984; 62: 1121-1133.

2. Possmayer F. The role of surfactant-associated proteins. Am Rev Respir Dis 1990; 142: 749-752.

3. Yamada T, Ikegami M, Jobe AH. Effects of surfactant subfractions on preterm rabbit lung function. Pediatr Res 1990; 27: 592-598.

4. Lewis JF, Veldhuizen R, Possmayer F, et al. Altered alveolar surfactant is an early marker of acute lung injury in septic adult sheep. Am J Respir Crit Care Med 1994; 150: 123-130. 
5. Malloy J, McCaig L, Veldhuizen R, et al. Alterations of the endogenous surfactant system in septic adult rats. Am J Respir Crit Care Med 1997; 156: 617-623.

6. Malloy J, Veldhuizen RAW, Lewis JF. Effects of ventilation on the surfactant system in sepsis-induced lung injury. J Appl Physiol 2000; 88: 401-408.

7. Sittipunt C, Steinberg KP, Ruzinski JT, et al. Nitric oxide and nitrotyrosine in the lungs of patients with acute respiratory distress syndrome. Am J Respir Crit Care Med 2001; 163: 503-510.

8. Arkovitz MS, Wispe JR, Garcia VF, Szabo C. Selective inhibition of the inducible isoform of nitric oxide synthase prevents pulmonary transvascular flux during acute endotoxemia. J Pediatr Surg 1996; 31: 1009-1015.

9. Turnage RH, Wright JK, Iglesias $\mathrm{J}$, et al. Intestinal reperfusion-induced pulmonary edema is related to increased pulmonary inducible nitric oxide synthase activity. Surgery 1998; 124: 457-462.

10. Laroux FS, Lefer DJ, Kawachi S, et al. Role of nitric oxide in the regulation of acute and chronic inflammation. Antioxid Redox Signal 2000; 2: 391-396.

11. Goad ME, Tryka AF, Witschi HP. Surfactant alterations following acute bleomycin and hyperoxiainduced lung damage. Toxicol Lett 1986; 32: 173-178.

12. Engstrom PC, Holm BA, Matalon S. Surfactant replacement attenuates the increase in alveolar permeability in hyperoxia. J Appl Physiol 1989; 67: 688693.

13. Gross NJ, Schultz RM. Requirements for extracellular metabolism of pulmonary surfactant: tentative identification of serine protease. Am J Physiol 1992; 262: L446-L453.

14. Ayad O, Wong HR. Nitric oxide decreases surfactant protein a gene expression in h441 cells. Crit Care Med 1998; 26: 1277-1282.

15. Laubach VE, Shesely EG, Smithies O, Sherman PA. Mice lacking inducible nitric oxide synthase are not resistant to lipopolysaccharide-induced death. Proc Natl Acad Sci U S A 1995; 92: 10688-10692.

16. Veldhuizen RA, Slutsky AS, Joseph M, McCaig L. Effects of mechanical ventilation of isolated mouse lungs on surfactant and inflammatory cytokines. Eur Respir J 2001; 17: 488-494.

17. Bligh EG, Dyer WJ. A rapid method of total lipid extraction and purification. Can J Biochem Physiol 1959; 37: 911-917.

18. Duck-Chong CG. A rapid sensitive method for determining phospholipid phosphorus involving digestion with magnesium nitrate. Lipids 1979; 14: 492 497.
19. Lowry OH, Rosebrough NJ, Farr AL, Randall RJ. Protein measurement with the Folin reagent. $J$ Biol Chem 1951; 193: 265-275.

20. Webert KE, Vanderzwan J, Duggan M, et al. Effects of inhaled nitric oxide in a rat model of Pseudomonas aeruginosa pneumonia. Crit Care Med 2000; 28: 2397 2405.

21. Rodriguez-Capote K, Nag K, Schurch S, Possmayer F. Surfactant protein interactions with neutral and acidic phospholipid films. Am J Physiol Lung Cell Mol Physiol 2001; 281: L231-L242.

22. Haddad IY, Pataki G, Hu P, Galliani C, Beckman JS, Matalon S. Quantitation of nitrotyrosine levels in lung sections of patients and animals with acute lung injury. J Clin Invest 1994; 94: 2407-2413.

23. De Sanctis GT, MacLean JA, Hamada K, et al. Contribution of nitric oxide synthases 1,2, and 3 to airway hyperresponsiveness and inflammation in a murine model of asthma. J Exp Med 1999; 189: 1621-1630.

24. Wang LF, Mehta S, Weicker S, et al. Relative contribution of hemopoietic and pulmonary parenchymal cells to lung inducible nitric oxide synthase (inos) activity in murine endotoxemia. Biochem Biophys Res Commun 2001; 283: 694-699.

25. Haddad IY, Zhu S, Ischiropoulos H, Matalon S. Nitration of surfactant protein A results in decreased ability to aggregate lipids. Am J Physiol 1996; 270: L281-L288.

26. Kobayashi H, Hataishi R, Mitsufuji H, et al. Antiinflammatory properties of inducible nitric oxide synthase in acute hyperoxic lung injury. Am J Respir Cell Mol Biol 2001; 24: 390-397.

27. Haddad IY, Zhu S, Crow J, Barefield E, Gadilhe T, Matalon S. Inhibition of alveolar type II cell ATP and surfactant synthesis by nitric oxide. Am J Physiol 1996; 270: L898-L906.

28. Veldhuizen RAW, Hearn SA, Lewis JF, Possmayer F. Surface-area cycling of different surfactant preparations: SP-A and SP-B are essential for large-aggregate integrity. Biochem J 1994; 300: 519-524.

29. Jobe A. Protein leaks and surfactant dysfunction in the pathogenesis of respiratory distress syndrome. Eur Respir J Suppl 1989; 3: 27s-32s.

30. Veldhuizen RA, McCaig LA, Akino T, Lewis JF. Pulmonary surfactant subfractions in patients with the acute respiratory distress syndrome. Am J Respir Crit Care Med 1995; 152: 1867-1871.

31. Gunther A, Siebert C, Schmidt R, et al. Surfactant alterations in severe pneumonia, acute respiratory distress syndrome, and cardiogenic lung edema. Am J Respir Crit Care Med 1996; 153: 176-184. 